\title{
Empirical Mode Decomposition and Blind Source Separation Methods for Antijamming with GPS Signals
}

\author{
Vinayak Kamath, Ying-Cheng Lai, Liqiang Zhu ${ }^{1}$ \\ Department of Electrical Engineering, Arizona State University, Tempe, AZ 85287, USA \\ Suprada Urval, \\ Qualcomm Inc., 2581 Junction Ave., San Jose, CA 95134, USA
}

\begin{abstract}
The spread-spectrum structure of GPS signals provides inherent jamming tolerance for GPS receivers. In a hostile environment where jamming sites may be close to GPS users, a larger JSR is possible. How to achieve the desired accuracy for GPS-based systems in the presence of strong jamming is an important but outstanding problem. Here we propose to use the empirical-mode decomposition (EMD) method, originally developed for analyzing nonlinear and nonstationary signals, for antijamming. Given a jammed, noisy GPS signal, the EMD method identifies the innate undulations belonging to different time scales and sifts them out to yield a small number of intrinsic modes. We find that the EMD method typically works well when the jamming is stationary in that the GPS signal and jamming components are typically contained in different intrinsic modes. However, when the jamming is nonstationary, the GPS signal and jamming are spread over all the intrinsic modes. Our solution is to use the blind-source separation (BSS) method operating on the set of intrinsic modes from EMD. Simulations indicated that this combined EMD/BSS methodology works reasonably well for extracting the GPS signal in the presence of nonstationary jamming for JSR up $45 \mathrm{~dB}$.
\end{abstract}

\section{INTRODUCTION}

The global positioning system (GPS) provides accurate three-dimensional positioning and timing information on any point on earth. The dependence of GPS for mission critical applications such as aircraft landing and military applications has increased steadily over the years. Good jamming excision techniques are therefore needed.

GPS signals are direct-sequence (DS) spread spectrum signals and therefore have some degree of inherent noise immunity (for eg, see [1]). After despreading the received signal, the original satellite signal can be collapsed into a narrow bandwidth about the carrier frequency, while the jamming signals are spread due to the lack of correlation with the pseudo-random code that encodes the satellite information. A portion of the spread jamming signal remains within the

\footnotetext{
${ }^{1}$ Contact address for Zhu: School of Mechanical, Electronic and Control Engineering, Beijing Jiaotong University, China
}

frequency band for the tracking loop with the satellite signal. Thus the signal tracking can be stable only if the jamming-tosignal ratio (JSR) is below the processing gain of the spreading code. In general, a JSR of greater than $30 \mathrm{~dB}$ is likely to prevent the GPS receiver from tracking the satellite signal and from estimating its own position.

There are situations where the jammer may be much stronger than the GPS signal, and are located at close physical proximity to the GPS receiver. In such a case, the spreading gain of the spread spectrum system might not be sufficient to decode the satellite data reliably. In fact, one of the major difficulties in time-space position information (TSPI) design in defense applications is jammer rejection. In this paper, we develop a procedure based on the empirical-mode decomposition (EMD) method [3], in combination with the traditional blind source separation (BSS) technique, as a receiver-based algorithm to suppress jamming. For practical implementation, our algorithm may be integrated into the software radio such as the one described in Ref. [7]. Schemes similar to ours have been suggested for applications in different contexts, such as biomedical signal processing, notably in Refs. [6] and [8] where a combination of EMD and independent component analysis was employed.

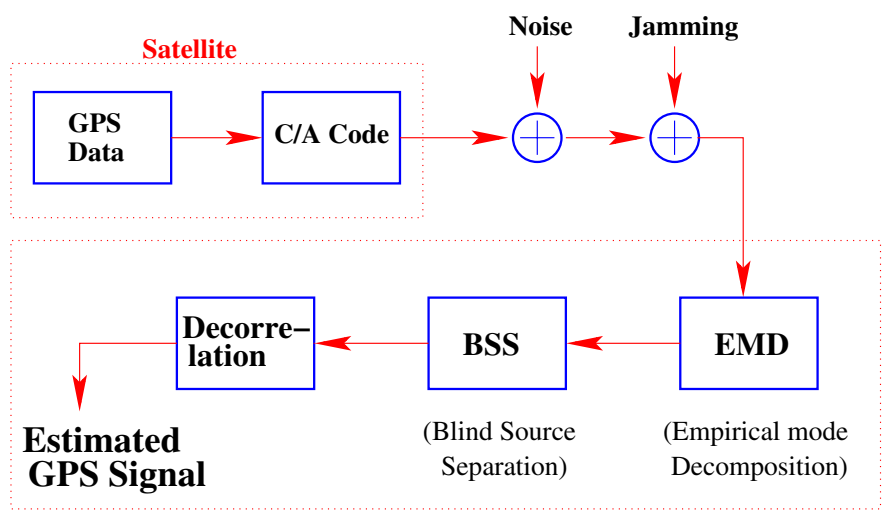

GPS Receiver

Fig. 1: Block Diagram of our proposed antijamming scheme 
The traditional Fourier analysis is powerful for stationary (or piecewise stationary) signals. The more recent wavelet method can handle nonstationary signals, but it is essentially an adjustable window Fourier spectral analysis and therefore fundamentally it is a linear method. The method of EMD was developed by Huang et al. to specifically deal with nonstationary and/or nonlinear signals [3]. That jamming signals in GPS applications are likely to be nonstationary and possibly nonlinear motivates us to investigate the applicability of EMD-based method for antijamming. The block diagram of our proposed method is shown in Fig. 1, where the original GPS data that carry the satellite information is encoded and both noise and jamming are present in the received GPS signal. The EMD/BSS combined procedure decomposes the jammed GPS signal into a number of modes of distinct time scales. Since the code that encodes the satellite information (the $\mathrm{C} / \mathrm{A}$ code) is also known at the receiver end, a decorrelation procedure can be used to identify the modes that contain mostly the original GPS data. We shall detail our method and provide strong evidence that the method works for nonstationary jamming of JSR ratio of up to $45 \mathrm{~dB}$.

In Sec. 2, we provide a brief background for the GPS signal structure and discuss several existing antijamming methods. In Sec. 3, we describe the EMD and BSS methods and their implementations. Simulation results with GPS signals are presented in Sec. 4. A discussion is presented in Sec. 5.

\section{Gps Signal Structure And Existing ANTIJAMMING METHODS}

GPS consists of 24 satellites orbiting at the altitude of $20,183 \mathrm{~km}$ at known positions. The signals transmitted by the GPS satellites are direct-sequence spread-spectrum (DSSS) signals that consist of three portions: the Course Acquisition (C/A) code on the L1 carrier $(1.575 \mathrm{GHz})$, the P-code on the $\mathrm{L} 1$ carrier, and the P-code on the L2 carrier $(1.227 \mathrm{GHz})$. The $\mathrm{C} / \mathrm{A}$ code has a chip-rate of $1.023 \mathrm{MHz}$ and a period of $1 \mathrm{msec}$, while the P-code, for military use only, has a chip-rate of $10.23 \mathrm{MHz}$ and a period of 1 week. As shown in Fig 2, with Binary Phase Shift Keying (BPSK) modulation, the resulting C/A-code signal requires a bandwidth of $2 \times 1.023 \mathrm{MHz}$ for transmission, and the P-code signal needs a bandwidth of $2 \times 10.23 \mathrm{MHz}$.

C/A Code

$1023000 \mathrm{~Hz}$

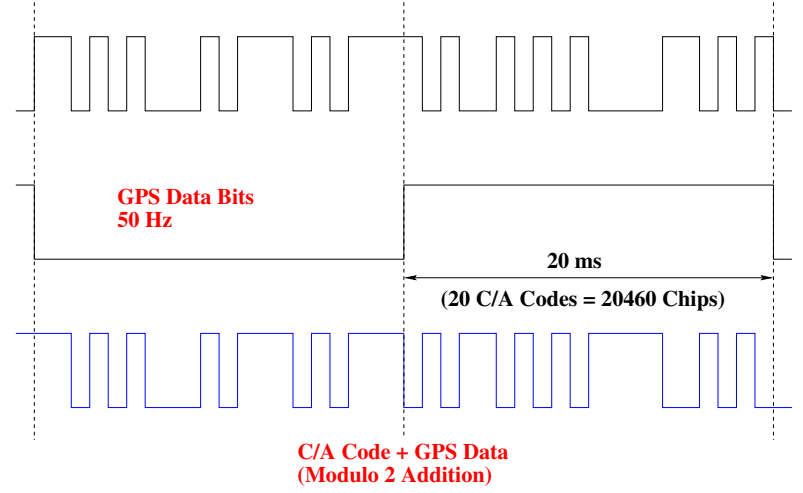

Fig. 2: GPS Direct Sequence spread-spectrum signal structure (C/A code)

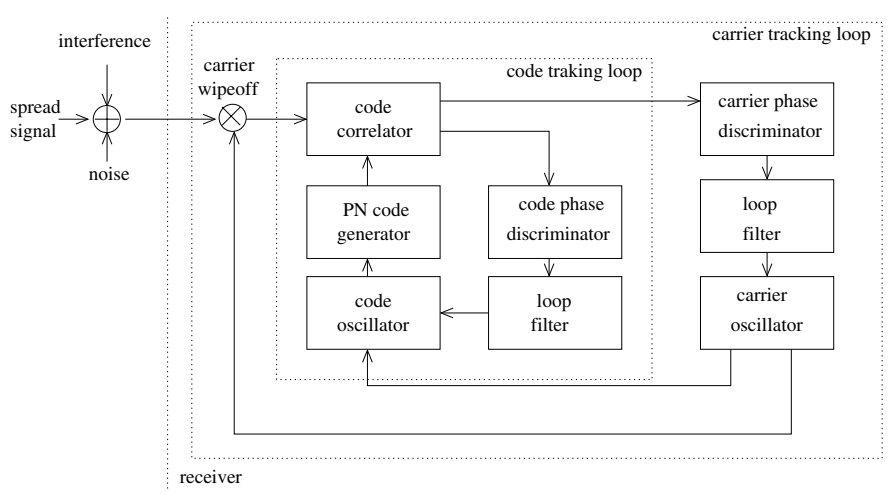

Fig. 3: Block Diagram of a GPS receiver that typically consists of a code and a carrier-phase tracking loop.

A GPS receiver receives DSSS signals from four or more satellites and estimates the code-phase differences and/or carrier-phase differences in order to calculate its own position [2]. As shown in Fig.3, the GPS receiver contains two tracking loops: code-phase tracking loop and carrier-phase tracking loop. The code tracking and despreading are performed prior to the carrier tracking since the power of the received GPS signal is much lower than the background noise and sufficient signal-to-noise ratio (SNR) necessary for carrier-phase tracking can only be achieved by code despreading. The pseudo-random noise $(\mathrm{PN})$ generator generates a PN sequence identical to the $\mathrm{C} / \mathrm{A}$ or the $\mathrm{P}$ code to synchronize with the code of the input signal from a GPS satellite. The code correlator sweeps the uncertainty ranges of the input code phase at discrete steps, and detects the coarse synchronization (acquisition) of the code phases between the input and local signals by finding the maximum of the correlation function. The code phase tracking loop can track the variations of the incoming code phase and keeps the codephase alignment error within an allowable limit after the code phase acquisition. From the accurate tracking of the code phase, the pseudo-range time delay can be obtained and the input signal is despread to obtain encoded navigation and timing information. The despread signal is then passed to the carrier-phase tracking loop for ranging.

In a hostile environment where jamming sites may be quite close to GPS users, a huge JSR is possible. Thus, in order for GPS receiver to function, jammer should be either rejected before the tracking loops or reduced/eliminated inside the tracking loops. For the first case, the structure of conventional tracking loops does not require change, while for the second case, novel structures need to be designed.

There are a number of ways to mitigate the effects of jamming on GPS receivers before the signal enters the tracking loops. These are (1) time domain filtering [19, 20], (2) frequency domain filtering $[17,18]$, (3) spatial filtering $[21,22]$, and (4) time-frequency filtering $[25,26]$. The first two types of filtering are conventional. The third type typically uses adaptive nulling antenna, an array of antenna elements. Spatial filters have the ability to modify its reception pattern, i.e., different amplification rates for signals from different directions of arrival (DOA). Based on the assumption that jamming signals and satellite signal have 
different DOA, adaptive nulling antenna can emphasize the desired GPS satellite signal and reject jamming signals [21]. The technique is effective for both narrow- and broad-band jamming signals. However, due to multi-path propagation of signals and the constraints on its size and power, the adaptive antenna alone cannot provide an acceptable interference mitigation. The fourth type (time-frequency filtering) relies on the assumption that broad-band satellite and jamming signals have distinct time-frequency signatures. Once the instantaneous frequencies of the jamming signals are estimated from the received signal, techniques such as timevarying notch filter [26] or subspace projection [25] can be used to reduce or eliminate the jamming. A possible drawback is that the time-frequency filter blocks the frequencies occupied by jamming signals, and thus also subtracts the power of satellite signal at those frequencies from the total power of the received signal. If jamming signals occupy a substantial bandwidth in the signal spectrum, the filtered signal will be significantly distorted. Thus, in general, this method is more effective for narrow-band jamming signals.

The conventional code tracking loop utilizes delay-lock loop (DLL), which has little protection against jamming and multipath. One way to increase the robustness of DLL is to include models (e.g., AR model) for jammer signal and multipath in the DLL. The resulting DLL can then estimate the code delay using adaptive algorithms, e.g., Kalman filters and/or particle filters [23, 24]

As we will describe, our proposed EMD/BSS procedure is fundamentally a nonlinear signal-processing method and it is therefore different from these existing linear methods.

\section{NONLINEAR ANTIJAMMING: Proposed EMd/BsS METHODOLOGY}

The method we propose applies EMD to the received jammed GPS signal to decompose it into a number of intrinsic mode functions (IMFs), which can be regarded as multiple observations of a random process. Assuming that these observations are linear combinations of the original signal, a BSS technique can be used to extract the GPS signal from the IMFs.

\section{A. $\quad$ EMD method}

Traditional methods such as the Fourier transform and spectral analysis assume stationarity and/or approximate the physical phenomena with linear models. These approximations may lead to spurious components in their time-frequency distribution diagrams if the underlying signal is nonstationary and nonlinear. Empirical Mode Decomposition (EMD) is a technique [3] to specifically deal with nonstationary and nonlinear signals. Given such a signal, the method adaptively decomposes it into a number of modes (IMFs) that are topologically equivalent to amplitude and frequency modulated, sinusoidal signals. In the analyticsignal representation, the modes correspond to proper rotations [3]. Thus the EMD method naturally yields estimates of the significant instantaneous frequencies embedded in the signal, by performing the Hilbert transform on each IMF. The ease and accuracy with which the EMD method processes nonstationary and nonlinear signals have led to its widespread use in various applications such as seismic data analysis [3], chaotic time series analysis [9, 10], neural signal processing in biomedical sciences [12], and meteorological data analysis [11], etc.

Given a signal $X(t)$, the EMD method focuses on the level of local oscillations and decomposes the signal into a finite and often a small number of fundamental oscillatory modes. The bases (IMFs) into which the signal is decomposed are obtained from the signal itself, and they are defined in the time domain. They are of the same length as the original signal and preserve the frequency variations with time. The base modes can be made approximately complete and nearly orthogonal with respect to each other. Here, completeness implies that the original signal can be reconstructed without any loss of data by simply adding up the IMFs. Thus, the IMFs can be viewed as linear components of the original or source signal $X(t)$. In order to achieve this, two conditions need to be satisfied: (1) the total number of extrema of $\operatorname{IMF}(t)$ be equal to the number of zero crossings, and (2) the mean of the upper envelope $I M F_{u}(t)$ and the lower envelope $I M F_{l}(t)$ be zero. The process to obtain the IMFs from the signal is called sifting, which consists of the following steps: (1) identification of the extrema of $X(t),(2)$ interpolation of the set of maximal and minimal points (say, by using cubic splines) to obtain an upper envelope $X_{u}(t)$ and a lower envelope $X_{l}(t)$, respectively, and their average $m(t) \equiv\left[X_{u}(t) \dashv X_{l}(t)\right] / 2$, (3) subtraction of the average from the original to yield $d(t)=X(t)-m(t)$, and (4) repetition of steps (1-3) until $d(t)$ satisfies the two conditions for being an IMF. Once an IMF is generated, the residual signal $r(t)=X(t)-I M F_{1}(t)$ is regarded as the original signal, and steps (1-4) are repeated to yield the second IMF, and so on. The procedure is complete when either the residual function becomes monotonic, or when the amplitude of the residue falls below a pre-determined small value so that further sifting would not yield any useful components. These features guarantee the computation of a finite number of IMFs within a finite number of iterations. The outcome of the EMD procedure is the following decomposition of the original signal:

$$
X(t)=\sum_{i=1}^{n} I M F_{i}(t)+r(t)
$$

where $I M F_{i}(t)$ is the ith IMF, $n$ is the total number of IMFs, and $r(t)$ is the final residue that has near zero 
amplitude and frequency.
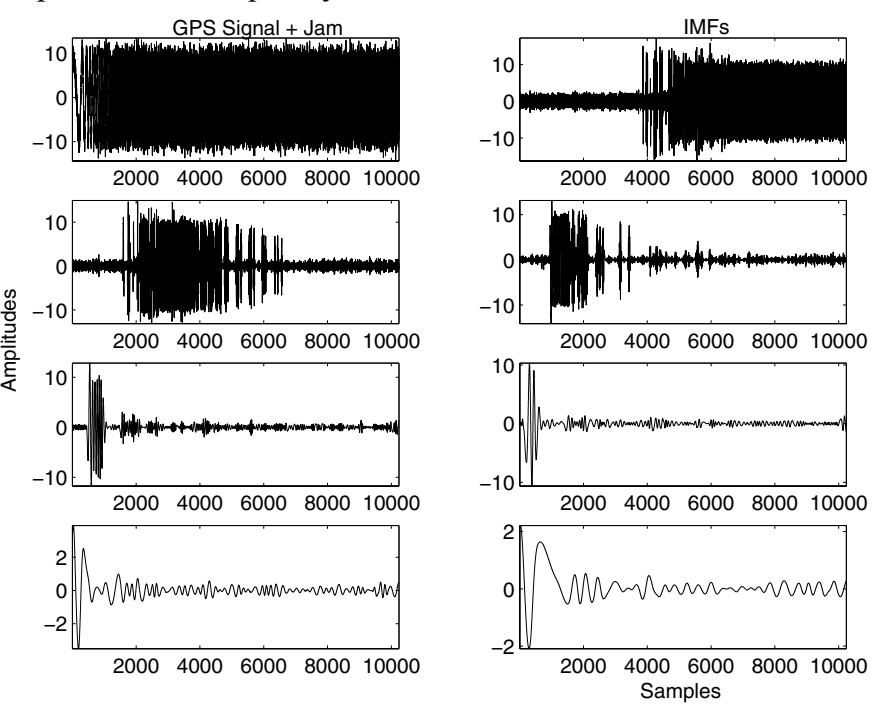

Fig. 4: IMFs of a noisy and jammed GPS signal, where the jamming is stationary and the noise is Gaussian. The first IMF (upper right panel) is approximately the original GPS signal.
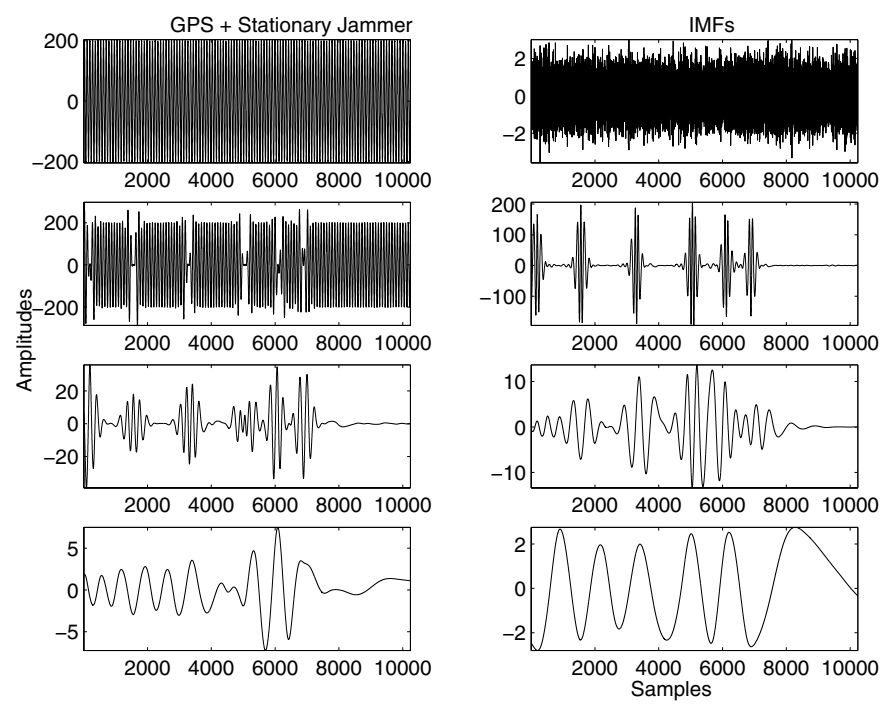

Fig. 5. IMFs of a noisy and jammed GPS signal, where the jamming is nonstationary and the noise is Gaussian. In this case, the original GPS signal is spread over all IMFs.

Fig. 4 shows the IMFs obtained from a simulated GPS signal to which a zero-mean and unit variance Gaussian noise and a stationary, sinusoidal jamming signal are added. The GPS signal has the amplitude of 2 and the jamming amplitude is 200 (corresponding to JSR of $45 \mathrm{~dB}$ ). The top left panel shows the noisy and jammed GPS signal, and the seven remaining panels show the seven significant IMFs. We note that the first IMF in the top right panel visually resembles the original GPS signal. This example thus illustrates that for stationary jamming, the EMD method is capable of directly separating the GPS signal from the jamming. However, this appears not to be the case for nonstationary jamming, as shown in Fig. 5, where the top left panel is the noisy and jammed GPS signal. The jamming is a frequency-modulated signal with normalized frequency increased linearly from 0 to 0.5 in the time interval considered. We find that none of the seven IMFs shown (in the remaining 7 panels) looks like the original GPS signal, which is in fact embedded in all IMFs. It is thus necessary to invoke a proper BSS procedure to extract the GPS signal.

\section{B. $\quad$ Blind Source Separation}

Blind Source Separation (see [13] for a review) is a method to extract basic source signals from several observed mixtures. It is considered "blind" because the source signals are not observed and no information is available about the observed mixtures. An a priori assumption is that the sources are independent of each other and they are not all white noise. BSS is particularly suitable for situations where different observations of the same sources are received from different sensors. The BSS technique has found applications in areas such as communications and biomedical signal processing [14, $15,16]$.

Consider the time varying vector of observations,

$$
\mathbf{X}(t)=\left[X_{1}(t), . ., X_{k}(t)\right]^{\prime}
$$

obtained as a mixture from $k$ sources

$$
\begin{gathered}
\mathbf{S}(t)=\left[S_{1}(t), . ., S_{k}(t)\right]^{\prime} \\
\mathbf{X}(t)=A \mathbf{S}(t),
\end{gathered}
$$

where A is the mixing matrix and ' denotes matrix transpose.

We reconstruct the sources

$$
\mathbf{Y}(t)=B \mathbf{X}(t)
$$

by adopting the criterion of minimizing mutual information. This requires the estimation of joint entropy which is computationally expensive, and therefore we adopt the idea of Gaussian Mutual information [4] which requires computation only up to second order characteristics. Using the instantaneous spectral density, $f_{Y_{k}}\left(t_{n}\right)$, of the process $Y_{k}(t)$ sampled at time $t_{n}$, the criterion for minimization of the Gaussian mutual information was shown in [5] to be

$$
C=\frac{1}{2} \sum_{k=1}^{K} \sum_{n=1}^{N} \sum_{m=1}^{M} \frac{1}{N M} \log \left[f_{y_{k}}\left(t_{n}, \lambda_{m}\right)\right]-\log \operatorname{det} \mathbf{B}
$$

where, the average instantaneous spectral density matrix $f_{Y_{k}}\left(t_{n}, \lambda_{m}\right)$ is obtained using a time frequency analysis window centered at $\left(t_{n}, \lambda_{m}\right)$ on the time-frequency plane.

\section{Simulation Results}

For simulation purposes, we use the following baseband model for received GPS signals:

$$
r(t)=c(t) d(t) \dashv j(t) \dashv n(t)
$$

where $r(t)$ is the received signal, $c(t)$ is the spreading 
sequence, $d(t)$ is the transmitted GPS information symbol, $n(t)$ is the noise, and $j(t)$ is the jamming. In the simulation, the length of spread sequence was varied from 10 to 20 bits. For stationary jamming simulations, pure sinusoids of frequency 0.2 to 0.5 times the sampling frequency were chosen. Nonstationary jamming was modeled as a chirp signal with frequency changing from 0 to 0.5 times the sampling frequency. The amplitude of the transmitted GPS signal is normalized to unity, while the jamming amplitude is varied so that the maximal JSR is $45 \mathrm{~dB}$.

The code for EMD was adapted from the one developed by Rilling et al. [Matlab codes (G. Rilling, P. Flandrin and P. Goncalves),http://perso.ens-lyon.fr/patrick.flandrin/emd.html]. During a run, typically between 8 and 10 IMFs are generated. As can be seen from the time series plots in Fig. 4, from the IMFs of stationary jammed GPS signal we can visually distinguish the jammer from the GPS signal. The GPS signal is captured almost entirely in the first mode, whereas the jammer is captured in the 2nd and 3rd modes. In this case, the BSS procedure is not necessary. To recover the GPS signal, it is a relatively simple matter of correlating the mode that contains the GPS signal with the PRN code. For nonstationary jammer, as in Fig. 5, both the GPS and the jamming are mixed in all the IMFs. In this case, it is necessary to use the BSS to extract the GPS signal. In particular, the IMFs can be regarded as multiple observations of the received signal. We adapted the code from the BLISS project [BSS Demonstration Code (http://www-lmc.imag.fr/SMS/SASI/bliss.html)] for BSS.

Fig. 6 shows the result of extracting the GPS signal in the presence of stationary jamming, where the two top panels show the original GPS and the original jamming signals,
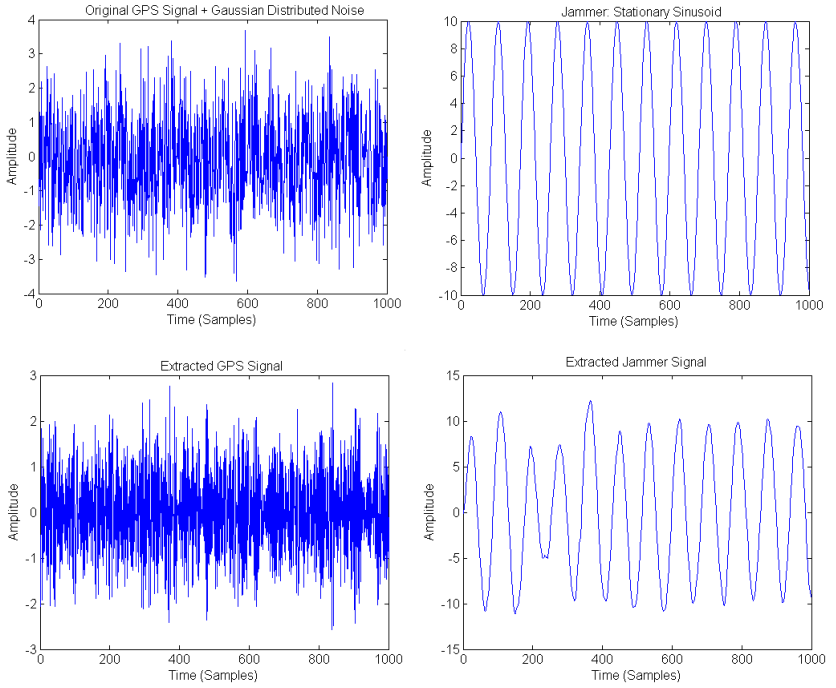

Fig. 6: Result with stationary jammer. Upper panels: original GPS signal and jammer. Lower panels: extracted GPS signal and jammer.
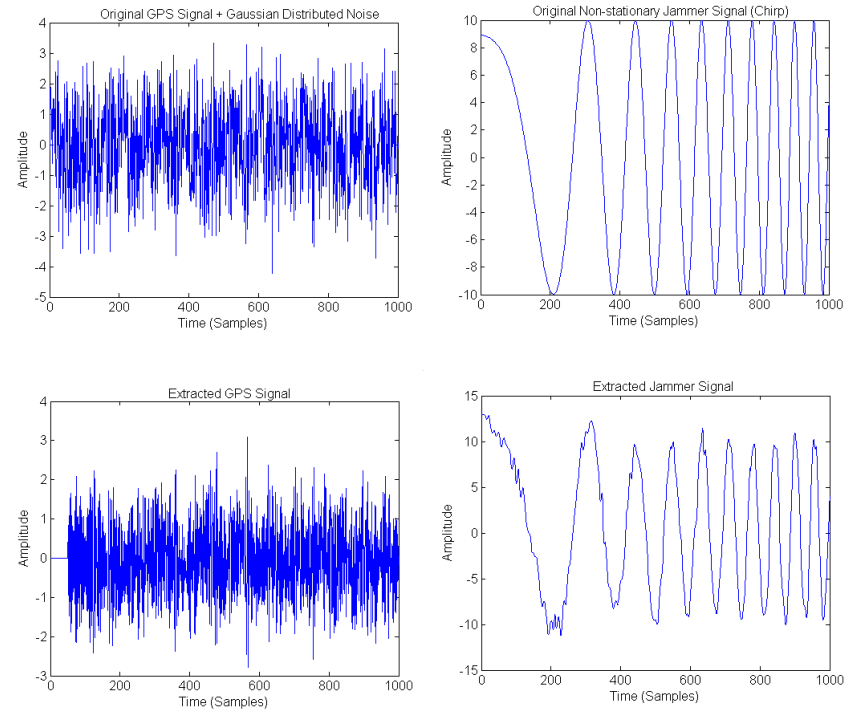

Fig. 7: Result with nonstationary jammer. Upper panels: original GPS signal and jammer. Lower panels: extracted GPS signal and jammer.

respectively, and the two lower panels show the extracted GPS and jamming signals. A typical result with nonstationary jamming is shown in Fig. 8. It is apparent that our combined EMD/BSS methodology is capable of estimating the GPS signal in the presence of strong jamming, stationary or nonstationary. To quantify the "goodness" of our method, we examine the bit error rate. In particular, the information bit associated with the extracted GPS signal is compared with that in the original GPS signal. A typical result for one C/A code is shown in Fig. 8, where the ratio of bits received correctly to the number of bits transmitted versus the jamming signal amplitude is plotted. The upper and lower traces show the ratio for stationary and nonstationary jamming, respectively. We see that for stationary jamming, the information bit can be extracted with certainty. For nonstationary jamming of JSR of as high as $45 \mathrm{~dB}$, the percentage of correctly extracted bit is above $80 \%$. Since one GPS bit ( $\mathbf{0}$ or $\mathbf{1}$ ) is modulated using $20 \mathrm{C} / \mathrm{A}$ codes, setting a conservative threshold, say at unity, for the ratio between the number of correctly estimated bits and that of the wrong bit can guarantee the extraction of the correct bit. In particular, suppose the original bit is 1 and the JSR is $40 \mathrm{~dB}$, then out of the $20 \mathrm{C} / \mathrm{A}$ codes, 1 will appear approximately 16 times and 0 (the wrong bit) will appear about 4 times. The ratio is then 4 , which can be distinguished from 1 almost certainly (in a probabilistic sense). Figure 8 suggests that our EMD/BSS method can perform to extract GPS satellite information in the presence of nonstationary jamming of JSR up to about $45 \mathrm{~dB}$.

\section{DISCUSSIONS}

Intentional jamming of GPS signals is a serious concern, especially for military applications. The nature of the GPS signal, i.e., its extreme low signal intensity, makes it highly 


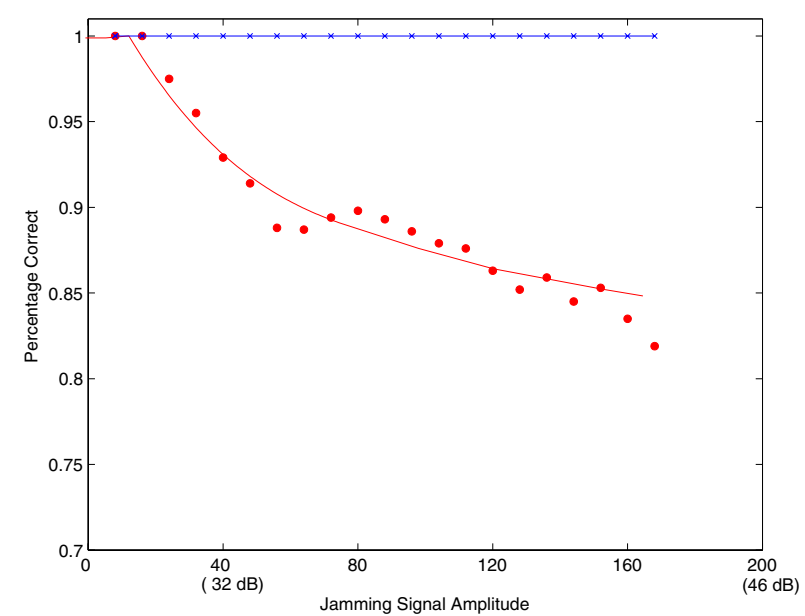

Fig. 8: For one C/A code, the percentage of correctly extracted number of GPS signal bits versus the jamming amplitude. The upper trace is for stationary jammer and the lower for nonstationary jammer. Since one GPS information bit is encoded using $20 \mathrm{C} / \mathrm{A}$ codes, for JSR of up to $45 \mathrm{~dB}$, the correct bit can be obtained with certainty.

vulnerable to jamming. In applications where dependence on GPS is high, a jammed GPS signal could have disastrous consequences. Thus it is of tremendous interest and importance to study practical antijamming schemes.

In this paper, we have proposed a simple yet effective antijamming algorithm which can be implemented in the receiver stage after the acquisition of the GPS signal. The algorithm makes use of two available techniques for data analysis: the Empirical Mode Decomposition method and the Blind Source Separation method based on Gaussian Mutual Information. We have shown that the algorithm is capable of accurate estimation of GPS signal bits in the presence of stationary or nonstationary jammers. In particular, simulation results indicate that the GPS data can be decoded accurately with no error for stationary jamming and nonstationary jammer of JSR of up to about $45 \mathrm{~dB}$.

For nonstationary jammer of JSR above $50 \mathrm{~dB}$, the performance of our method deteriorates. The solution may be to combine this algorithm with spatial filtering methods such as those described in [21, 22]. Another possibility is to integrate Kalman or particle filtering in the algorithm for estimating the phase/code delay. The model for such a system would assume the presence of jamming interference and, when combined with our algorithm, might enable GPS signal processing in the presence of exceedingly strong jammer.

\section{ACKNOWLEDGMENT}

This work was supported by AFOSR under Grant No. FA9550-04-1-0115.

\section{REFERENCES}

[1] S. Haykin, Communication Systems, 4th ed., Wiley, New York, 2001

[2] B. Hofmann-Wellenhof, H. Lichtenegger, and J. Collins, Global Positioning System, Theory and Practice, 5th ed., Springer-Verlag, New York, 2001
[3] N.E. Huang, et al, "The empirical mode decomposition and the Hilbert spectrum for nonlinear and non-stationary time series analysis," Proc. R. Soc. A, vol. 454, pp. 903-995, 1998.

[4] D.T. Pham, "Blind Separation of Instantaneous Mixture of Sources via an Independent Component Analysis," IEEE Trans. Sig. Proc. vol. 44, pp. 2768$2779,1996$.

[5] D.T. Pham, "Exploiting source non stationary and coloration in blind source separation," 14th Int. Conf. DSP, vol. 1, pp. 151-154, 2002.

[6] M.J. McKeown, R. Saab, and R. Abu-Gharbieh, "A combined independent component analysis (ICA)/empirical mode decomposition (EMD) method to infer corticomuscular coupling". Neural Engineering, Proc. Int. Conf. IEEE EMBS, pp. 679-682, 2005.

[7] J.B.Y. Tsui, Fundamentals of GPS Receivers: A Software Approach, 2nd Ed, John Wiley and Sons, New York, 2005.

[8] R. Balocchi, D. Menicucci, and M. Varanini, "Empirical mode decomposition to approach the problem of detecting sources from a reduced number of mixtures," Proc. Int. Conf. IEEE EMBS, 2003.

[9] L. Yalcinkaya, and Y-C. Lai, "Phase characterization of chaos," Phys. Rev. Lett., vol. 79, pp. 3885-3888, 1997.

[10] Y-C Lai, "Analytic signals and transition to chaos in deterministic flows,” Phys. Rev. E, vol. 58, pp. R6911-R6914, 1998.

[11] D.G. Duffy, "The application of Hilbert-Huang transforms to meteorological datasets," J. Atmos. Oceanic Tech, vol. 21, pp. 599-611, 2004.

[12] H. Liang, Q. Lin, and J.D.Z. Chen, "Application of the empirical mode decomposition to the analysis of esophageal manometric data in gastroesophageal reflux disease," IEEE Trans. Biomed. Eng, vol. 52, pp. 1692-1701, 2005.

[13] A. Mansour, A.K. Barros, and N. Ohnishi, "Blind separation of sources: Methods, assumptions and applications," IEICE Trans. Funda. Electronics, Commun. Comp. Sci., vol. E83-A, pp. 1498-1512, 2000.

[14] K. Anand, G. Mathew, and V. U. Reddy, "Blind separation of multiple co-channel BPSK signals arriving at an antenna array," IEEE Sig. Proc. Lett., vol. 2, pp. 176-178, 1995.

[15] E. Chaumette, P. Comon, and D. Muller, "An ICA-based technique for radiating sources estimation: application to airport surveillance," IEE Proc. Part F, vol. 140, pp. 395-401, 1993.

[16] D.L. Lathauwer, D.B. Moor, and J. Vandewalle, "Fetal electrocardiogram extraction by source subspace separation," Proc. of IEEE Sig. Proc., pp. 134-138, 1995.

[17] B. Badke, and A. Spanias, "Partial band interference excisions for GPS using frequency-domain exponents," Proc. of IEEE Conf Acoustics, Speech, and Signal Processing 13-17 May 2002 :IV-3936--IV-3939, 2002.

[18] P.T. Capozza, B.J. Holland, T.M. Hopkinson, and R.L. Landrau, "A single-chip narrow-band frequency-domain excisor for a GPS receiver," IEEE J. Solid State Circuits, vol. 35, pp. 401-411, 2000

[19] J.W. Ketchum, and J.G. Proakis, "Adaptive algorithms for estimating and suppressing narrow-band interference in PN spread-spectrum systems," IEEE Trans. Comm., vol. 30, pp. 913-924, 1982.

[20] L.B. Milstein, "Interference rejection techniques in spread spectrum communications," Proc. IEEE, vol. 76, pp. 657-671, 1988

[21] R. Fante R, and J.J Vaccaro, "Wideband cancellation of interference in a GPS receiver array," IEEE Trans. Aerospace Elec. Sys., vol. 36, pp. 549-564, 2000 . 
[22] W.L. Myrick, J.S. Goldstein, M.D. Zoltowski, "Low complexity antijam space-time processing for GPS," Proc. IEEE Inter. Conf. Acoustic, Speech, and Signal Processing, vol. 4, pp. 2233-2236, 2001.

[23] R.A. Iltis, "Joint estimation of PN code delay and multipath using the extended Kalman filter," IEEE Trans. Comm., vol. 38, pp. 1677-1685, 1990.

[24] S.J. Kim, R.A. Iltis, "Performance comparison of particle and extended kalman filter algorithms for GPS C/A code tracking and interference rejection," Proc. of Conf. Information Sciences and Systems, 2002.

[25] M.G. Amin, R.S. Ramineni, A.R. Lindsey, "Suppression of FM interferences in DSSS communications using projection techniques," Proc. of the 33rd ASILOMAR conference, 1999.

[26] M.G. Amin, "Interference mitigation in spread-spectrum communication systems using time-frequency distributions," IEEE Trans. Sig. Proc., vol. 45, pp. 90-102, 1997. 